\title{
Investigación documental sobre el cuerpo y la corporeidad en la escuela
}

\author{
Documentary Research on Body and Corporeity at School \\ Pesquisa documental sobre o corpo e a corporalidade na escola
}

Recibido • Received • Recebido: 29 / 10 / 2019

Corregido • Revised $\cdot$ Revisado: 18 / 07 / 2021

Aceptado • Accepted • Aprovado: 12 / 08 / 2021

\begin{abstract}
Resumen: Esta investigación documental sintetiza la producción científica realizada entre los años 20132018 acerca de los significados de cuerpo y corporeidad en el contexto escolar y en otros contextos. Las referencias provienen de las bases de datos Scopus, Red de Repositorios Latinoamericanos de la Universidad de Chile y repositorios online de ocho bibliotecas de universidades chilenas. Las búsquedas se hacen en las áreas de ciencias sociales, artes y humanidades y psicología, a través de los descriptores: Corpo, Body, Cuerpo y Educación, Cuerpo y Educación Física, Corporalidad; Corporeidad e imagen Corporal, Asessment and Conceptions and Representations. Fueron seleccionados 100 artículos de Scopus y de los repositorios se capturaron 152 documentos. Los hallazgos demuestran 7 focos de interés investigativo referidos a: estudiar el cuerpo en el contexto escolar, el abordaje teórico-metodológico, una línea en progreso de acercamiento a la dimensión subjetiva del cuerpo, prácticas corporales fuera de la escuela, representación del cuerpo en los medios, la mirada sociocultural del cuerpo y, finalmente, el estudio sobre sexo, género y cuerpo. En conclusión, en las referencias internacionales prevalece el paradigma positivista, con una concepción biomédica que emana desde diversos contextos; en las referencias latinoamericanas prevalece el estudio cuerpo asociados a la escuela, aunque desde una mirada transversal se observan indicios de generar conocimiento sobre la dimensión corporal subjetiva y expresiva. Aún falta investigar y escuchar la diversidad de las personas como actores sociales, en vistas de potenciales mejoras en las políticas educativas protectoras de la dignidad y justicia social de los cuerpos.
\end{abstract}

Palabras claves: Análisis cualitativo, corporeidad; cuerpo; educación física; escuela; investigación documental; investigación pedagógica. 
http://doi.org/10.15359/ree.25-3.31

http://www.una.ac.cr/educare

educare@una.ac.cr

\begin{abstract}
This documentary research synthesizes the scientific production carried out between 2013 and 2018 about the meanings of body and corporeity in the school context and other contexts. The references come from the Scopus databases, the Latin American Repositories Network of the University of Chile, and the online repositories of eight Chilean University Libraries. The searches were conducted in the areas of social sciences, arts and humanities, and psychology through the following descriptors: corpo, body, cuerpo and educación, cuerpo and educación física, corporalidad, corporeidad and imagen corporal, assessment, and conceptions and representations. One hundred Scopus articles were selected, and 152 documents were captured from the repositories. The findings showed seven focuses of research interest referring to studying the body in the school context, the theoreticalmethodological approach, a line in the progress of approach to the subjective dimension of the body, body practices outside of school, representation of the body in the media, the sociocultural view of the body, and finally, the study of sex, gender, and the body. In conclusion, in international references, the positivist paradigm prevails with a biomedical conception that emanates from different contexts. In the Latin American references, the body study associated with the school prevails; although, from a transversal view, there are indications of generating knowledge about the subjective and expressive dimension of the body. It is still necessary to investigate listening to the diversity of people as social actors in view of potential improvements in educational policies that protect the dignity and social justice of the bodies.
\end{abstract}

Keywords: Qualitative analysis; corporeity; body; physical education; school; documentary research; pedagogical investigation.

Resumo: Esta pesquisa documental sintetiza a produção científica realizada entre os anos 20132018, sobre os significados de corpo e corporeidade no contexto escolar e em outros contextos. As referências provêm das bases de dados Scopus, da Rede Latino-americana de Repositórios da Universidade do Chile e dos repositórios online de oito Bibliotecas Universitárias chilenas. As pesquisas são feitas nas áreas de Ciências Sociais, Artes e Humanidades e Psicologia, por meio dos descritores: Corpo, Body, Corpo e Educação, Corpo e Educação Física, Corporalidade; Corporeidade e Imagem Corporal, Assessment and Conceptions and Representations. Foram selecionados 100 artigos de Scopus e dos repositórios foram selecionados 152 documentos. As descobertas demonstram 7 focos de interesse de pesquisa referentes a: estudo do corpo no contexto escolar, abordagem teórico-metodológica, linha permanente de abordagem da dimensão subjetiva do corpo, práticas corporais fora da escola, representação do corpo na mídia, a visão sociocultural do corpo e, por fim, o estudo do sexo, gênero e corpo. Em conclusão, nas referências internacionais prevalece o paradigma positivista, com uma concepção biomédica que emana de diferentes contextos; nas referências latino-americanas, prevalece o estudo do corpo associado à escola; embora do ponto de vista transversal haja indícios de geração de conhecimento sobre a dimensão subjetiva e expressiva do corpo. Ainda é necessário investigar a escuta da diversidade das pessoas como atores sociais, tendo em vista possíveis melhorias nas políticas educacionais que protejam a dignidade e a justiça social dos corpos.

Palavras-chave: Análise qualitativa; corporalidade; corpo; educação física; escola; pesquisa documental; pesquisa pedagógica. 


\section{Introducción}

La investigación documental o bibliográfica es un método crucial en el abordaje y comprensión profunda del contexto histórico, espacial y temporal en que se revela una problemática específica. Hernández Sampieri et al. (2014) plantean que este tipo de investigación consiste en: "detectar, consultar y obtener la bibliografía (referencias) y otros materiales que sean útiles para los propósitos del estudio, de donde se tiene que extraer y recopilar la información relevante y necesaria para enmarcar nuestro problema de investigación" (p.61). Estas referencias emanan de diversas fuentes documentales como tesis, libros, revistas, apuntes de investigación, actas, artículos, entre otras (Chong de la Cruz, 2007; Rojas Rojas, 2007; Rojas Crotte, 2011).

La revisión bibliográfica o construción del estado del arte (Guevara Patiño, 2016) es una de las etapas fundamentales en la producción investigativa, teórico-metodológica y de análisis crítico acerca de un tema (Hernández Sampieri et al., 2014; Pantoja Villarreal, 2006), la que es útil para aproximarse el estado de avance en que se encuentra una investigación, conocer lo que se ha hecho en torno a la temática, qué falta por hacer, reconocer tendencias, autores y autoras principales y, así, aportar o profundizar en diversas áreas de conocimiento sin redundar en el conocimiento ya existente. Igualmente, ayuda a clarificar ideas respecto al tema de interés, definir, afianzar o modificar objetivos y orientar la línea de investigación. En otras palabras, es la configuración del estado del arte, que es "seguirle las huellas a un proceso hasta identificar su estado de desarrollo más avanzado" (Londoño Palacio et al., 2016, p. 5).

El origen de esta metodología data de fines de la década de los setenta y principios de los ochenta, cuando se dirigieron fundamentalmente estudios en el área de las ciencias sociales en América Latina, específicamente en Colombia, donde se trataba de recopilar y compilar la información disponible por distintas vías de forma simultánea sobre un tema en particular (Molina Montoya, 2005), que hasta el día de hoy ha evolucionado y adquirido un enfoque científico, siendo utilizada por investigaciones de diversa índole, tanto desde una perspectiva cuantitativa como cualitativa.

Dado que este concepto es relativamente reciente, se pueden encontrar distintas definiciones que intentan esclarecer el término; no obstante, todas coinciden en que se trata de un "tipo de evaluación descriptiva; evaluación seria, sistematizada y consistente. Supone el revivir de una mínima parte de la memoria científica de la humanidad en aquel campo dentro del cual enmarcamos nuestro proyecto investigativo" (Bojacá Acosta, 2004, p. 193).

Este proceso se desarrolla en una fase heurística de búsqueda basada en criterios de inclusión y de sistematización de las fuentes informativas; luego avanza a una fase hermenéutica de lectura, análisis, interpretación y clasificación, conforme a la importancia interna que cobra en la pesquisa (Rojas Rojas, 2007). Es en el proceso de sistematización donde se toman en cuenta los datos para su futuro análisis, tal como el rango de años en la producción investigativa, temáticas de interés, metodologías abordadas, principales personas autoras, el tipo de recurso bibliográfico 
http://doi.org/10.15359/ree.25-3.31

http://www.una.ac.cr/educare

educare@una.ac.cr

encontrado, entre otros. Sin embargo, en el proceso de búsqueda de información no basta solo con enlistar y describir sobre el fenómeno; también se busca alcanzar un juicio crítico y desarrollar cierto nivel de comprensión y conexión que se tiene sobre la temática, una reflexión profunda de los alcances y vacíos que puedan existir, tal como lo indica Hoyos Botero (2000):

El proceso... nada dice si no es en íntima conexión con el otro eje de esa unidad dialéctica: el colectivo de investigadores. Este remite indudablemente a un equipo comprometido, donde lo teórico, lo práctico y lo contextual se conjuguen con el ejercicio de profundos y reconocidos valores éticos. (p. 15)

Si bien existe gran alcance a la información por medio de las nuevas tecnologías, dicho alcance podría ser hasta abrumador para el equipo investigador si no se tiene claridad del objeto y las áreas de conocimiento a las que quiere apuntar. En el área de Educación, en particular en el área de la Educación Física escolar, las experiencias internacionales muestran que los esfuerzos investigativos han sido abordados principalmente desde el área de las ciencias de la vida: medicina, bioquímica, genética, biología molecular, neurociencia y afines, donde ha prevalecido un enfoque dualista cartesiano respecto a la funcionalidad del cuerpo (González Plate, 2014). Pero ¿qué hay de los esfuerzos en las ciencias sociales y humanidades, las artes y la psicología? ¿Cuáles son las principales temáticas investigadas? ¿Qué paradigma investigativo prevalece? ¿Serán abordadas desde los agentes sociales que interactúan con y en la escuela? ¿Existe aún la dominación del dualismo cartesiano en el quehacer que se desarrolla en la escuela?, entre muchas otras interrogantes que se pueden formular.

En el caso del proceso de búsqueda de información correspondiente al presente artículo, que forma parte del proyecto ANID-Fondeyt №11170199: La corporeidad en la escuela: Un reflejo de su esencia y presencia en nuestra sociedad, se interesa en conocer lo publicado a este respecto a nivel internacional, latinoamericano y particularmente en Chile. Para este abordaje se deja de lado la razón biológica, anatómica y fisiológica de concebir el cuerpo, para hacer una revisión desde el prisma de las ciencias sociales y humanidades; en vistas de fortalecer el desarrollo teórico-metodológico comprensivo, evidenciar la productividad científica, los contextos y los enfoques desde los cuales se estudia el cuerpo y las corporeidades. El estudio se circunscribe, en una primera etapa, a un diseño cualitativo e interpretativo de tipo documental, donde se trata de conocer, describir y analizar críticamente, a partir de la información encontrada en los datos y no en las teorías previas, permitiendo dar cuenta de información detallada y descriptiva sobre las principales temáticas (Gómez Vargas et al., 2015), asociadas a la dimensión corporal.

A partir de las evidencias sistematizadas se busca revelar un panorama del estado de desarrollo de lo investigado (Londoño Palacio et al., 2016; Gómez Vargas et al., 2015), así como las metodologías empleadas, los contextos abordados y las personas investigadoras más destacadas. Al profundizar en el conocimiento y dar lectura a los documentos encontrados, se puede adentrar en el análisis concluyente donde florecen categorías en relación con la 
corporeidad y motricidad de los diversos agentes sociales que interactúan con y en la escuela; lo cual se podría comprender como un reflejo de la cultura de la sociedad latinoamericana en relación a los sentidos sociales y culturales que se le otorgan al cuerpo y las corporeidades, tal como lo plantean Gubbay y Kalmar (1990, citados por Blanco-Vega, 2011, p. 5):

La Expresión Corporal nace de y valoriza aquella manifestación corporal espontánea existente desde siempre, pues nuestra forma de existencia es corporal. El cuerpo constantemente se manifiesta desde su concepción hasta el momento de su muerte. Se va modelando según las experiencias vividas dentro del contexto familiar, social y cultural. Es el modo de internalizar el mundo y responderle.

Desde esta perspectiva cabe preguntarse ¿desde qué enfoques del cuerpo y corporeidades se posesionan las propias personas investigadoras para sumergirse en el estudio de lo que investigan? ¿Qué podrán decir los investigadores e investigadoras en el área de educación física y de las ciencias de la educación sobre la existencia corporal? ¿Qué enseñanzas dejan los estudios sobre el cuerpo en las etnias? ¿Cómo influyen los medios en las visiones sobre el cuerpo? ¿Qué representaciones existen sobre sexo y género asociados al cuerpo? ¿Las referencias estudiadas serán las suficientes para avanzar en la resignificación de la corporeidad en el quehacer formativo de la educación escolar en general y en la educación física en particular? Se podrá repensar una reforma educativa (Morin, 2007) y ponerla en marcha desde un currículo integrador y desarrollador de saberes con el de la propia vida (Arias y Peralta, 2011; Gómez Francisco, 2018), donde el reconocimiento de la dimensión corporal no pueda quedar excluido, dado que:

No hay nada que podamos decir, sentir, pensar o desear, en lo que no esté implicado nuestro cuerpo y, a su vez, no hay concepción de cuerpo que no esté atravesada por el lenguaje y la cultura y, así pues, lo cultural, social, simbólico y discursivo se materializan 'en'y'a través' del cuerpo. En esta medida, hablar sobre el cuerpo es hablar corporalmente. Sentir es sentirnos a nosotros mismos, de tal modo que nuestra relación con nosotros mismos, con los otros y con las cosas solo es posible a través de nuestra corporalidad (Gallo Cadavid (2009, p. 232).

Dicho lo anterior, se abre una invitación a la lectura y nuevas reflexiones sobre lo que se ha hecho y lo que aún falta por hacer.

\section{Desarrollo}

La metodología empleada para la elaboración del estado de desarrollo de la temática estudiada se establece sobre diversas bases de datos de producción internacional y latinoamericana. Cabe mencionar que, conforme a los intereses investigativos de las autoras, también se hace una búsqueda particularizada de la realidad chilena, en vistas a poder determinar las temáticas estudiadas y el acercamiento metodológico (Tabla 1). 
http://doi.org/10.15359/ree.25-3.31

http://www.una.ac.cr/educare

educare@una.ac.cr

Tabla 1: Búsqueda para la construcción del estado del arte

\begin{tabular}{|c|c|c|c|c|}
\hline & Fuente deinformación & Recurso bibliográfico & $\begin{array}{l}\text { N. }{ }^{\circ} \text { de publicaciones } \\
\text { categorizadas }\end{array}$ & N. ${ }^{\circ}$ de categorías \\
\hline \multirow{3}{*}{ Estado del arte } & Scopus & Solo artículos científicos & 100 artículos & 16 categorías \\
\hline & $\begin{array}{l}\text { Red Latinoamericana de } \\
\text { Repositorios }\end{array}$ & $\begin{array}{l}\text { Tesis, libros, actas, } \\
\text { revisión, papers }\end{array}$ & 94 publicaciones & 11 categorías \\
\hline & $\begin{array}{l}\text { Repositorio de } \\
\text { universidades a nivel } \\
\text { nacional }\end{array}$ & $\begin{array}{l}\text { Tesis, proyectos } \\
\text { investigativos, libros solo } \\
\text { de producción nacional }\end{array}$ & 58 publicaciones & 11 categorías \\
\hline
\end{tabular}

Nota: Elaboración propia.

En el proceso de búsqueda internacional se hace uso de la base de datos de Scopus, aplicando los descriptores de: Cuerpo and Corpo*; Body and Corpo*; Body or Corpo* (el asterisco fue utilizado para incluir en la búsqueda las palabras "corporeidad" y "corporalidad" simultáneamente), así como Asessment and Conceptions and Representations; estos últimos en inglés para corroborar coincidencias o bien, tener un mayor sesgo de información científica. Los criterios de selección de los recursos bibliográficos seleccionados se concentran en solo artículos científicos, publicados entre los años 2013 al 2018, en idiomas inglés, español y portugués, provenientes de diversos países, asociados a las áreas de conocimiento de las Ciencias Sociales, Artes y Humanidades y Psicología.

Dentro de estas búsquedas se encontró un total de 43.057 artículos; no obstante, diversos escritos se encontraban en más de un área de conocimiento. Por consiguiente, se seleccionaron por su contenido, referido a las concepciones, significados, valoraciones, representaciones e imaginarios del cuerpo y la corporalidad, arrojando como resultado 100 artículos, los que fueron copiados en un documento Word para ser analizados bajo una lectura de sus respectivos resúmenes y, a partir de esto, levantar 16 categorías de forma manual.

Para el proceso de búsqueda a nivel latinoamericano, se recurre al portal de la Red de Repositorios Latinoamericanos, desarrollado por la Universidad de Chile (UCH) con más de 1,3 millones de publicaciones académicas de acceso abierto, con más de 90 instituciones, entre universidades, organismos gubernamentales y no gubernamentales de 19 países. Los recursos bibliográficos seleccionados dentro de este repositorio se concentran en tesis, libros, actas de congreso, papers, y revisiones de acceso abierto, preferentemente en idioma español; dentro de estas búsquedas se reunió un total de 94 publicaciones, pertenecientes a diversas áreas de conocimiento, seleccionadas por su contenido referido a las concepciones, significados, valoraciones, representaciones e imaginarios del cuerpo y la corporalidad, las que fueron copiadas en un segundo documento Word, aparte de la búsqueda internacional, analizadas de igual forma que en dicho repositorio. Se levantó un total de 11 categorías de forma manual. 
Finalmente, dentro del repositorio a nivel nacional, se consideran las referencias chilenas publicadas en la Red de Repositorios Latinoamericanos de la Universidad de Chile antes mencionada, junto a la revisión de las bibliotecas online de 12 universidades tradicionales chilenas, que son de interés para nuestro proyecto Fondecyt, tales como la Pontificia Universidad Católica de Valparaíso, la Universidad de Playa Ancha, la Universidad de Tarapacá, la Universidad Metropolitana de Ciencias de la Educación, la Universidad Católica del Maule, la Universidad de Concepción, la Universidad de la Frontera y la Universidad Católica Silva Henríquez. A objeto de ampliar la búsqueda, se consideran otras instituciones, tales como la Universidad de Valparaíso, la Universidad Técnica Federico Santa María, la Universidad Adolfo lbáñez y la Pontificia Universidad Católica de Chile.

Los recursos bibliográficos seleccionados fueron libros de producción nacional, tesis, proyectos investigativos y publicaciones periódicas en papel. Los descriptores afines utilizados para la búsqueda fueron: Corpo, Cuerpo y Educación, Cuerpo y Educación Física, Corporalidad; Corporeidad e Imagen Corporal. En el caso de los libros que aportan las diversas universidades, se incluyen dentro de la categorización solo aquellos de producción nacional, por autores chilenos o bien, que abordan temáticas culturales desarrolladas en Chile.

Dentro de los documentos encontrados, se agregan a un tercer listado general para ser categorizado según el tema que trata, de igual manera que en la búsqueda de información anterior, es decir, se copian en un documento Word los títulos, personas autoras, tipo de recurso bibliográfico y resúmenes de cada texto, dejando un resultado de 58 documentos, los cuales finalmente fueron categorizados de forma manual bajo 11 temáticas, de igual forma que en el repositorio de nivel latinoamericano.

\section{Resultados}

Tomando en consideración en su conjunto el proceso de búsqueda de las referencias publicadas en las bases de datos mencionadas, en el período comprendido entre 2013 y 2018, se logra reunir 252 publicaciones. De ellas, 179 se asocian con el área de conocimiento de las Ciencias Sociales y Humanidades, 61 con el área de Artes y Humanidades y 12 con el área de Psicología. Del proceso interpretativo realizado emergen 17 grandes categorías: 8 asociadas a las Ciencias Sociales, 6 categorías en Artes y Humanidades y 3 categorías en el área de Psicología (Tabla 2).

En relación con los resultados específicos, se puede distinguir que de los 100 artículos seleccionados dentro de Scopus predomina claramente la producción de las ciencias sociales por sobre las artes y humanidades, y aún en mayor medida en comparación con la psicología. En términos cuantitativos, el $77 \%$ de los artículos pertenece a las ciencias sociales, $20 \%$ a las artes y humanidades y $3 \%$ de los artículos al área de psicología. 
http://doi.org/10.15359/ree.25-3.31

http://www.una.ac.cr/educare

educare@una.ac.cr

Tabla 2: Resultados de búsqueda y sistematización asociados a Scopus

\begin{tabular}{|c|c|c|c|c|}
\hline & & Área de conocimiento & Principales autores & Categorías \\
\hline \multirow{3}{*}{ SCOPUS } & \multirow{3}{*}{$\begin{array}{c}100 \text { artículos } \\
\text { científicos }\end{array}$} & Ciencias Sociales 77\% & \multirow{3}{*}{$\begin{array}{l}\text { Da Nóbrega, T. P. (3, Brasil) } \\
\text { Almeida, F. Q. (2, Brasil) } \\
\text { Herold Junior, C. (2, Brasil) }\end{array}$} & $\begin{array}{l}\text { - Satisfacción corporal (27) } \\
\text { - Abordaje teórico-metodológico sobre cuerpo y } \\
\text { corporalidades (17) } \\
\text { - Afectaciones/Vulneraciones sobre el cuerpo (9) } \\
\text { - Cuerpo en la escuela (9) } \\
\text { - Educación del cuerpo (8) } \\
\text { - Sensaciones corporales (4) } \\
\text { - Representaciones corporales desde los docentes EFI (3) }\end{array}$ \\
\hline & & Artes y Humanidades $20 \%$ & & $\begin{array}{l}\text { - Representación de cuerpo en los medios (5) } \\
\text { - Cuerpo y etnia (4) } \\
\text { - Cuerpo y religión (4) } \\
\text { - Prácticas corporales fuera de la escuela (4) } \\
\text { - Cuerpo y comunicación (2) } \\
\text { - Cuerpo en la historia (1) }\end{array}$ \\
\hline & & Psicología 3\% & & $\begin{array}{l}\text { - Concepciones desde las personas psicólogas (1) } \\
\text { - Cuerpo según el contexto (1) } \\
\text { - Percepciones sobre el sexo (1) }\end{array}$ \\
\hline
\end{tabular}

Nota: Elaboración propia.

Asimismo, se aprecia una tendencia al idioma inglés en las publicaciones, a pesar de que gran parte de las personas autoras provienen de países donde el principal idioma es el español o el portugués.

Entre las personas autoras por países más nombradas, se evidencia que Brasil aporta 7 publicaciones originadas por Da Nóbrega, T. P., con 3 publicaciones, seguido por Almeida, F. Q. y Herold Junior, C., con 2 publicaciones cada uno. Luego el aporte proviene de España, con Pozo, J., y Reino Unido, con Longo, M. R.; cada uno de ellos con 2 publicaciones respectivamente.

En relación con la frecuencia de aparición de las categorías, existe una predominancia, en orden descendente, en relación con la Satisfacción corporal, Abordaje teórico-metodológico sobre cuerpo y corporalidades y Afectaciones/Vulneraciones sobre el cuerpo. Por otra parte, las categorías con menor frecuencia de aparición corresponden a cuerpo según el contexto, cuerpo en la historia, concepciones desde las personas psicólogas y percepciones sobre el sexo, con 1 aparición cada una.

Con respecto a la búsqueda específica de lo que se está produciendo en la región Latinoamérica, sin considerar para este análisis las referencias chilenas según lo señalado anteriormente, la mayor producción proviene del área de conocimiento de las Ciencias Sociales: del total de 94 publicaciones, esta aporta el 69,1\% de la producción; luego se evidencia el aporte del área de las Artes y Humanidades con un 28,7\% y el área de Psicología con el 2,1\%. Ver tabla 3. 
http://doi.org/10.15359/ree.25-3.31

http://www.una.ac.cr/educare educare@una.ac.cr

Tabla 3: Resultados de búsqueda y sistematización asociado a la Red Latinoamericana de Repositorios

\begin{tabular}{|c|c|c|c|c|}
\hline & & $\begin{array}{c}\text { Área de } \\
\text { conocimiento }\end{array}$ & Principales personas autoras & Categorías \\
\hline ha & & $\begin{array}{l}\text { Ciencias Sociales } \\
69,1 \%\end{array}$ & $\begin{array}{l}\text { Emiliozzi, M. V. (5, Argentina) } \\
\text { Galak, E. (5, Argentina) }\end{array}$ & $\begin{array}{l}\text { - Educación desde el cuerpo (21) } \\
\text { - Abordaje teórico-metodológico sobre cuerpo y } \\
\text { corporalidades (20) } \\
\text { - Cuerpo en el currículo (10) } \\
\text { - Representaciones corporales desde el personal } \\
\text { docente EFI (8) } \\
\text { - Cuerpo en la escuela (6) }\end{array}$ \\
\hline & & $\begin{array}{l}\text { Artes y Humanidades } \\
28,7 \%\end{array}$ & $\begin{array}{l}\text { Patierno, N. (2, Argentina) } \\
\text { Ferrarese, S. M. (2, Argentina) }\end{array}$ & $\begin{array}{l}\text { - Representación de cuerpo en los medios (11) } \\
\text { - Prácticas corporales fuera de la escuela (7) } \\
\text { - Cuerpo en la historia (7) } \\
\text { - Cuerpo y etnia (2) }\end{array}$ \\
\hline & & $\begin{array}{l}\text { Psicología } \\
2,1 \%\end{array}$ & & $\begin{array}{l}\text { - Imagen corporal (1) } \\
\text { - Sexo, género y cuerpo (1) }\end{array}$ \\
\hline
\end{tabular}

Nota: Elaboración propia.

Entre las personas autoras por países más nombradas, se evidencia que Argentina aporta 14 publicaciones originadas principalmente por Emiliozzi, M. V., con 5 apariciones en diversas categorías como en educación del cuerpo, cuerpo en el currículum y representación del cuerpo en los medios. De igual forma le sigue Galak, E., con 5 apariciones entre categorías como educación del cuerpo, cuerpo en la escuela, representaciones corporales desde los docentes de educación física. Otras personas autoras que también figuran son Patierno, N. y Ferrarese, S. M., cada uno con 2 publicaciones respectivamente; finalmente, Napolitano, M. E., con 1 publicación en colaboración con Galak, E.

En relación con la frecuencia de aparición de las categorías, existe una predominancia, en orden descendente, en relación con educación desde el cuerpo, abordaje teórico-metodológico sobre cuerpo y corporalidades y cuerpo en el currículo. Por otra parte, las categorías con menor frecuencia de aparición corresponden a imagen corporal y sexo, género y cuerpo, con 1 aparición cada una.

Es a nivel latinoamericano donde se comienzan a vislumbrar en mayor medida las categorías referidas al cuerpo y la corporalidad asociadas a los agentes sociales que interactúan con la escuela; a diferencia del nivel internacional, donde predominan los enfoques biométricos y patológicos del cuerpo.

En cuanto a la búsqueda específica de producción chilena que se encuentra publicada en el repositorio latinoamericano y en las bases de datos online de ocho bibliotecas de las universidades chilenas seleccionadas para fines del presente estudio, se puede evidenciar 
http://doi.org/10.15359/ree.25-3.31

http://www.una.ac.cr/educare

educare@una.ac.cr

que la mayoría proviene del área de conocimiento de las Ciencias Sociales: del total de las 58 publicaciones seleccionadas, aporta el 63,8\%. Luego vienen las artes y humanidades con un 24,1\%, y el área de Psicología con el 12,1\% (Tabla 4).

Tabla 4: Resultados de búsqueda y sistematización asociados al repositorio

\begin{tabular}{|c|c|c|c|c|}
\hline & & Área de conocimiento & Principales personas autoras & Categorías \\
\hline \multirow{3}{*}{ Repositorio Nacional } & \multirow{3}{*}{58 textos } & Ciencias sociales $63,8 \%$ & \multirow{3}{*}{$\begin{array}{l}\text { Autores diferentes, no se } \\
\text { identifica uno que lidere }\end{array}$} & $\begin{array}{l}\text { - Educación desde el cuerpo (11) } \\
\text { - Cuerpo en la escuela (11) } \\
\text { - Cuerpo en el currículo (6) } \\
\text { - Representaciones corporales desde el } \\
\text { personal docente EFI (6) } \\
\text { - Abordaje teórico-metodológico sobre } \\
\text { cuerpo y corporalidades (3) }\end{array}$ \\
\hline & & Artes y humanidades $24,1 \%$ & & $\begin{array}{l}\text { - Prácticas corporales fuera de la escuela (5) } \\
\text { - Cuerpo en la historia (4) } \\
\text { - Cuerpo y etnia (3) } \\
\text { - Cuerpo y religión (2) }\end{array}$ \\
\hline & & Psicología 12,1\% & & $\begin{array}{l}\text { - Sexo, género y cuerpo (6) } \\
\text { - Representaciones sobre cuerpo y salud } \\
\text { alimentaria (1) }\end{array}$ \\
\hline
\end{tabular}

Nota: Elaboración propia.

Entre las personas autoras más nombradas, no se aprecia que prevalezca alguna en relación con la cantidad de publicaciones, lo que conduce a pensar que no existe una línea investigativa consolidada al respecto. En este caso, todos los documentos se encuentran en idioma español.

En relación con la frecuencia de aparición de las categorías, lideran la educación desde el cuerpo, cuerpo en la escuela, y sexo, género y cuerpo, en contraste con representaciones sobre cuerpo y salud alimentaria, con solo una aparición.

Finalmente, se puede señalar que la categoría de Educación desde el cuerpo se hace presente dentro del repositorio latinoamericano y chileno. Puede abarcar desde conceptos como educación corporal, educación física, motricidad, y educación psicomotriz; sin embargo, no necesariamente se relacionan con la corporalidad desde una concepción monista y subjetiva, sino que también lo hacen desde una concepción dualista del cuerpo, que enfatiza en la funcionalidad y rendimiento como un cuerpo-máquina. Hallazgo que conlleva a la obtención de resultados y pistas para la discusión y conclusiones. Este proceso consistió en la contextualización y constatación de los hallazgos alcanzados a través de la categorización, para finalmente elaborar un informe narrativo e interpretativo a partir de la documentación encontrada. 


\section{Análisis}

Cuando de cuerpo se habla en las referencias científicas, generalmente se encuentran asociadas al área de conocimiento de las Ciencias de la Vida, particularmente desde las Ciencias Médicas que generalmente asumen una perspectiva biológica, medible, objetiva y cuantificable. Sin embargo, desde búsquedas realizadas en las áreas de las ciencias sociales y humanas, de las artes y humanidades y de la psicología, se abre un abanico de posibilidades temáticas de desarrollo de conocimiento en torno al cuerpo y la corporalidad; así también, muestra la forma en que es concebido por los actores y personas autoras, ya sea que provengan de los medios de comunicación; profesionales de la salud, de la educación, docentes en formación; las prácticas deportivas u otros tipos de prácticas corporales fuera de la escuela, actores religiosos, etnias, entre otros. Aunque no necesariamente estos grupos se planteen desde un enfoque de cuerpo como la presencia encarnada, en la perspectiva de Merleau-Ponty (1994); presencia que percibe al mundo y que se expresa desde la interacción consigo misma, las cosas y los demás, como el ser pensante, sensible y perceptivo en y desde la corporalidad.

Al analizar en su conjunto las 8 categorías temáticas que emergen del área de Ciencias Sociales, 3 categorías con 82 publicaciones tienen como principal objeto de estudio el cuerpo y la corporeidad en el contexto de la escuela, referidas a: educación desde el cuerpo (40), cuerpo en el currículo (16) y cuerpo en la escuela (26); de las cuales 65 documentos tienen mayor presencia en los escritos encontrados en el repositorio latinoamericano y bases de datos de universidades chilenas.

A modo de aclarar el proceso analítico llevado a cabo, las categorías que han sido mencionadas referían a los siguientes criterios:

- Educación desde el cuerpo: Involucra ambos abordajes de la educación corporal en la escuela, ya sea como una educación corporal integradora de saberes, y expresiones motrices, así como la EFI como reproductora de patrones motrices, con enfoque biomédico.

- Cuerpo en la escuela: Refiere al abordaje de cuerpo y corporalidad en la escuela tradicional, sin concentrarse en el quehacer de la clase de Educación Física.

- Cuerpo en el currículo: Aborda el concepto de cuerpo en planes de estudios, estructuras curriculares, metodologías, planificaciones y didácticas.

El segundo foco de estudio corresponde al abordaje teórico-metodológico sobre cuerpo y corporalidades, que concita el interés de 40 de las publicaciones. el tercer foco de interés investigativo se puede sintetizar como una línea de acercamiento a la dimensión subjetiva del cuerpo con 57 documentos que atienden a: representaciones corporales desde los docentes de educación física (17), sensaciones corporales (4), satisfacción corporal (27) y afectaciones/ vulneraciones sobre el cuerpo (9). Se evidencia que ambos focos de interés sobresalen en las búsquedas de Scopus y del repositorio latinoamericano. 
http://doi.org/10.15359/ree.25-3.31

http://www.una.ac.cr/educare

educare@una.ac.cr

En tanto, en el área de conocimiento de las artes y humanidades, se observan 6 temáticas de estudio, con 61 publicaciones; especialmente surgen de Scopus y del repositorio latinoamericano. Los dos primeros focos de interés son: prácticas corporales fuera de la escuela (16) y representación de cuerpo en los medios (16); el tercer foco de estudio se ha centrado en la cultura y el cuerpo con temáticas sobre cuerpo e historia (12), etnia (9), religión (6) y comunicación (2).

En el área de psicología, se evidencian 3 categorías con 12 publicaciones, las cuales tienen como principal objeto de estudio el sexo, género y cuerpo (8) y se muestra como el principal foco e interés en el repositorio chileno. El segundo objeto se concentra en el estudio de las representaciones e imagen corporal (4) que se localizan en Scopus y en el repositorio latinoamericano.

En este caso, la documentación recogida es un reflejo de la calidad de las investigaciones que se publican: la producción chilena es menor en comparación con países como España, Reino Unido, Argentina, Brasil y Colombia. Ahora bien, el debate entre las concepciones dualista y monista (Le Breton, 2002; Puglisi, 2014) aún permanece en las produccciones científicas; no en todas se articulan el cuerpo y la escuela, especialmente en lo referido a la dimensión subjetiva del cuerpo que reconoce a la persona humana como sujeto encarnado y su expresión gestual (Merleau-Ponty, 1994).

\section{Conclusiones}

Las referencias internacionales antes comentadas emanan principalmente de la opción teórico-metodológica que se ampara en el paradigma positivista y que pone el acento en una concepción biomédica del cuerpo. En Latinoamérica, líderes en publicaciones en relación con el cuerpo y la corporalidad corresponden a Argentina, Brasil y Colombia, y en su mayoría corresponden a abordajes teóricos-metodológicos del cuerpo asociados con la escuela. Pero, pese a llevar el concepto de escuela entre sus palabras claves, no necesariamente se ligan al estudio sobre el trato subjetivo que las instituciones educativas les dan a las corporeidades.

La baja cobertura de publicaciones en este mismo sentido podría atribuirse al aún presente dualismo cartesiano, particularmente por el arraigado enfoque de la Educación Física centrado en la higiene, salubridad, productividad y mejoramiento de la raza (Matus Zapata, 1932), que ha sido fuertemente influenciado por las escuelas y sistemas de la gimnasia alemana y sueca del siglo XIX y principios del siglo XX y que ha dejado huella en los países de Latinoamérica (Lara Díaz, 2003; Poblete Gálvez et al., 2014). Hoy en día la educación ha evolucionado; no obstante, en la formación inicial docente y en el sistema educativo escolar chileno aún permanecen pensamientos de aquella época que se enfocan en la funcionalidad y rendimiento del cuerpo (González Plate, 2014; Moreno Doña et al., 2014), dejando de lado aspectos lúdicos, sensoriales, 
http://doi.org/10.15359/ree.25-3.31

expresivos, rítmicos, de toma de conciencia, entre otros. En palabras de Martínez Álvarez y García Monge (1997): "Hay un olvido generalizado de la naturaleza corpórea de la educación. Por ello, paradójicamente, la manifestación más significativa de la presencia del cuerpo en la escuela es su ignorancia o relegación" (p. 174). Se suman a lo anterior los actuales argumentos acerca del impacto de los crecientes índices de obesidad en la población.

Es indudable que estos aspectos han incidido en la baja producción investigativa sobre el cuerpo subjetivo o encarnado (Merleau-Ponty, 1994); particularmente en las experiencias vividas en el acto de aprender en la escuela. Esto implica que la perspectiva de la corporeidad debe ser considerada como una construcción social, en la cual se logren enfocar, analizar y comprender las características, semejanzas y diferencias que pueda poseer cada persona. Es de esa individualidad que nos diferencia como humanos, que nos hace ser únicos dentro del aspecto social, dentro de un mundo diverso; no obstante, dicha diversidad plantea retos al sistema educativo en su conjunto, puesto que, tal como lo expresa Gimeno Sacristán (2000):

Plantear el problema de la diversidad y de la diferencia en y ante la educación supone enfrentarse con retos y ámbitos de significados muy polivalentes: la lucha contra las desigualdades, el problema de la escuela única interclasista, la crisis de los valores y del conocimiento tenidos por universales, las respuestas ante la multiculturalidad y la integración de minorías (p. 69).

Pensamos que para contribuir en el cambio del enfoque del cuerpo, se hace necesario ponerlo en la discusión social pero también en la discusión científica; falta investigar la corporeidad desde la diversidad de las personas como actores sociales, de las formas de sentirse, pensarse y ser tratadas corporalmente, en vistas de potenciales mejoras en las políticas educativas en favor de la dignidad y justicia social (Moreno Doña y Poblete Gálvez, 2015); para así generar mayor alcance a la información, publicar y revisar el material que ofrecen las revistas científicas y repositorios universitarios para asegurar la calidad la información de la ciencia que se realiza, no solo en la Educación Física, sino también para lograr mayores exigencias en la disciplina y mejor preparación de los profesionales que trabajan en ella.

Finalmente, cabe denotar que al hacer una lectura transversal de los principales focos de estudio que se desprenden del conjunto de publicaciones se observan indicios de generación de conocimiento sobre la dimensión subjetiva y expresividad corporal que alcanzan mayor presencia en los repositorios latinoamericanos, lo que se convierte en una oportunidad para seguir profundizando y establecer redes con las principales autores y autoras que se han mencionado de Argentina, Brasil y Colombia. Esto significa un gran desafío e invitación para elaborar más investigaciones al respecto, que sirvan como antecedentes para establecer políticas que permitan impulsar una Educación más humanizadora del cuerpo y de su reconocimiento como una dimensión presente en la formación que se vive en la escuela. 
http://doi.org/10.15359/ree.25-3.31

http://www.una.ac.cr/educare

educare@una.ac.cr

\section{Declaración de procedencia y financiamiento}

Este artículo se ha realizado gracias al apoyo de la Agencia Nacional de Investigación y Desarrollo (ANID, Chile) que, a través Fondo Nacional de Desarrollo Científico y Tecnológico (FONDECYT) Iniciación, ha financiado el proyecto N. ${ }^{\circ} 11170199$, titulado "La corporeidad en la escuela: Un reflejo de su esencia y presencia en nuestra sociedad".

\section{Declaración de Material complementario}

Este artículo tiene disponible, como material complementario:

-La versión preprint del artículo en https://doi.org/10.5281/zenodo.4067061

\section{Referencias}

Arias, R. y Peralta, H. (2011). La enseñanza, una puerta para la complejidad y la crítica. Estudios pedagógicos, 37(1), 293-302. https://dx.doi.org/10.4067/S0718-07052011000100017

Blanco Vega, M. de J. (2011). La expresión corporal en la formación de maestros: Estudio de los programas de educación inicial en las universidades de Bogotá. Revista Q. Educación Comunicación Tecnología, 5(10), 1-20. https://dialnet.unirioja.es/ejemplar/309492

Bojacá Acosta, J. (2004). XYZ investigación pedagógica estado del arte semilleros. Logos-edit.

Chong de la Cruz, I. (2007). Métodos y técnicas de la investigación documental. En H. A. Figueroa Alcántara y C. A. Ramírez Velázquez (coords.), Investigación y docencia en bibliotecología (pp. 183-201). Facultad de Filosofía y Letras, UNAM. http://ru.ffyl.unam.mx/bitstream/ handle/10391/4716/12 IDB 2007_ I_Chong.pdf?sequence=1\&isAllowed=y

Gallo Cadavid, L. E. (2009). El cuerpo en la educación da qué pensar: Perspectivas hacia una educación corporal. Estudios Pedagógicos, 35(2), 232-242. https://dx.doi.org/10.4067/ $\underline{\text { S0718-07052009000200013 }}$

Gimeno Sacristán, J. (2000). La educación obligatoria: Su sentido educativo y social. Morata.

Gómez Francisco, T. (2018). La complejidad: Un paradigma para la educación. Su aporte con una mirada histórica y reflexiva. RIL editores; Universidad de Atacama. http://www.salud.uda. cl/wp-content/uploads/2020/04/La-complejidad.pdf 
Gómez Vargas, M., Galeano Higuita, C. y Jaramillo Muñoz, D. A. (2015). El estado del arte: Una metodología de investigación. Revista Colombiana de Ciencias Sociales, 6(2), 423-442. https://doi.org/10.21501/22161201.1469

González Plate, L. (2014). Representaciones sociales de los y las estudiantes de pedagogía sobre la educación física escolar en Chile [Tesis de doctorado]. Universidad de Granada. https:// digibug.ugr.es/handle/10481/34704

Guevara Patiño, R. (2016). El estado del arte en la investigación: ¿Análisis de los conocimientos acumulados o indagación por nuevos sentidos? Folios, (44), 165-179. https://www.redalyc. org/pdf/3459/345945922011.pdf

Hernández Sampieri, R., Fernández-Collado, C. y Baptista Lucio, P. (2014). Metodología de la investigación (6. ${ }^{a}$ ed.). McGraw-Hill. https://bit.ly/3bohzFW

Hoyos Botero, C. (2000). Un modelo para investigación documental: Guía teórico-práctica sobre construcción de estados del arte con importantes reflexiones sobre la investigación. Señal Editora.

Lara Díaz, H. (2003). Gimnasia. Documento de apoyo a la docencia. Departamento de Educación Física, Deportes y Recreación. Universidad Metropolitana de Ciencias de la Educación.

Le Breton, D. (2002). Antropología del cuerpo y modernidad. Ediciones Nueva Visión.

Londoño Palacio, O. L., Maldonado Granados, L. F. y Calderón Villafáñez, L. C. (2016). Guías para construir estados del arte. ICONK.

MartínezÁlvarez, L.y García Monge,A. (1997). Educación física y género. Una mirada al cuerpo en la escuela. En Alario Trigueros, T. y García Colmenares, C. (coords.), Persona, géneroy educación (pp. 31-71). Amarú Ediciones. https://www.researchgate.net/publication/263730939 Educacion Fisica y genero Una mirada al cuerpo en la escuela

Matus Zapata, L. (1932). El problema de la educación física en el Chile. El embrujamiento sueco. Imprenta y Encuadernación La República.

Merleau-Ponty, M. (1994). Fenomenología de la percepción. Península. https://monoskop.org/ images/9/9b/Merleau-Ponty Maurice_Fenomenologia_de_la_percepcion_1993.pdf

Molina Montoya, N. P. (2005). ¿Qué es el estado del arte? Ciencia y Tecnología para la Salud Visual y Ocular, 3(5), 73-75. https://doi.org/10.19052/sv.1666

Moreno Doña, A., Gamboa Jiménez, R. y Poblete Gálvez, C. (2014). La educación física en Chile: Análisis crítico de la documentación ministerial. Revista Brasileira de Ciências do Esporte, 36(2), 411-427. http://www.redalyc.org/pdf/4013/401334038010.pdf 
http://doi.org/10.15359/ree.25-3.31

http://www.una.ac.cr/educare

educare@una.ac.cr

Moreno Doña, A. y Poblete Gálvez, C. (2015). La educación física chilena y su profesorado: Proponiendo algunos retos para la investigación en el área. Retos, 28, 291-296. https://doi. org/10.47197/retos.v0i28.35651

Morin, E. (2007). La cabeza bien puesta: Repensar la reforma. Repensar el pensamiento. Nueva Visión. https://www.academia.edu/37337267/La_cabeza_bien_puesta_Edgar_Morin_1

Pantoja Villareal, M. I. (2006). Construyendo el objeto de estudio e investigando lo investigado: Aplicaciones de un estado del arte. Revista Memorias, 4(8), 104-107. https://jceducacion. files.wordpress.com/2011/06/construyendo-el-objeto-de-estudio.pdf

Poblete Gálvez, C., Moreno Doña, A. y Rivera García, E. (2014). Educación Física en Chile: Una historia de la disciplina en los escritos de la primera publicación oficial del Instituto de Educación Física de la Universidad de Chile (1934-1962). Estudios pedagógicos, 40(2), 265282. https://dx.doi.org/10.4067/S0718-070520140000300016

Puglisi, R. (2014). Repensando el debate monismo versus dualismo en la antropología del cuerpo. Cuadernos de Antropología Social, (40), 73-95. https://www.redalyc.org/articulo. oa?id=180938244004

Rojas Crotte, I. R. (2011). Elementos para el diseño de técnicas de investigación: Una propuesta de definiciones y procedimientos en la investigación científica. Tiempo de Educar, 12(24), 277-297. http://www.redalyc.org/pdf/311/31121089006.pdf

Rojas Rojas, S. P. (2007). El estado del arte como estrategia de formación en la investigación. Studiositas, 2(3), 5-14. https://dialnet.unirioja.es/servlet/articulo?codigo=2719676 